\title{
PERCEPÇÃO DE VALOR EM DISPOSITIVOS TECNOLÓGICOS GERADORES DE INFORMAÇÕES ALIMENTARES DE PRODUTOS ALIMENTÍCIOS CUSTOMIZADOS
}

Luiz Philipi Calegari (luizpcalegari@gmail.com) - Departamento de Pós gradução em Engenharia de Produção e Sistemas, Universidade Federal de Santa Catarina.

Diego Castro Fettermann (dcfetterman@gmail.com) - Departamento de Pós gradução em Engenharia de Produção e Sistemas, Universidade Federal de Santa Catarina.

Giuliano Zandonai (giuzandonai@gmail.com) - Departamento de Engenharia de Produção e Sistemas, Universidade Federal de Santa Catarina.

\section{RESUMO}

Em produtos alimentícios, a disponibilidade de informações sobre o produto (rótulos por exemplo), influencia perante a percepção de valor. Mas, à medida que o produto alimentício é customizado, a personalização dessas informações torna-se necessária, fato este que pode criar dificuldades na adoção de Customização em Massa (CM). A utilização de dispositivos que usufruem de tecnologia inteligente (smart technology) é um meio capaz de auxiliar os consumidores no momento da escolha dos produtos alimentícios, e possibilitam a customização de informações. A partir de uma pesquisa de mercado sobre dispositivos que geram informações sobre produtos alimentícios, foram identificados 5 atributos considerados na composição desses dispositivos: (i) portabilidade, (ii) precisão, (iii) personalização de dieta, (iv) análise de qualidade do produto alimentício e (v) preço. Este estudo possui como objetivo, identificar quais desses atributos direcionam para uma maior agregação de valor ao cliente. Realizou-se então um projeto experimental desenvolvido por análise conjunta baseada em escolha, com planejamento fatorial fracionado $2^{5-1}$. Para a coleta de dados foi realizada a abordagem metodológica survey, com número de 130 respondentes. Como contribuições, este estudo traz resultados para o direcionamento da pesquisa para composição de um dispositivo com finalidade de fornecer informações sobre o produto alimentício customizado em massa.

Palavras chave: customização em massa; restrições alimentares; personalização; informações alimentares; dispositivos tecnológicos; percepção de valor

Área: Open Innovation

\section{INTRODUÇÃO}

A identificação da necessidade do consumidor e a interação dessa percepção com a manufatura pode ser considerada uma barreira para a implementação da Customização em Massa (CM) (DA SILVEIRA et al., 2001). O processo de reconhecimento da necessidade do consumidor está relacionado com o período de pré-compra, que depende da capacidade dos consumidores de perceber e processar informações relacionadas ao produto (DIMARA; SKURAS, 2005). Desta forma, a cooperação do cliente durante o processo de desenvolvimento do produto torna-se imprescindível para adoção de CM (DURAY et al., 2000). A utilização de mecanismos facilitadores para comunicação entre cliente e empresa, com a finalidade de traduzir as necessidades individuais dos consumidores em especificações 
de um produto (DA SILVEIRA et al., 2001; FETTERMANN et al., 2012), são considerados um dos principais determinantes para o sucesso de adoção de CM (OGAWA; PILLER, 2006).

O processamento das informações contidas nos rótulos dos produtos consiste em uma importante fonte de dados para o cliente decidir sobre a compra do produto (KRIFLIK; YEATMAN, 2005). Apesar disso, a forma como os consumidores processam as informações presentes nos rótulos dos produtos para sua tomada de decisão ainda não é consensual na literatura (VAN DER MERWE et al., 2010), assim como o efeito negativo da falta de informações sobre o alimento em seu rótulo (LINDLEY, 2007). Estudos apontam que influência dos rótulos na decisão de compra estão associados a fatores relacionados com sua confecção, tais como cor de rotulagem (SCHULDT, 2013), presença de elementos simbólicos, tais como emoctions (VASILJEVIC et al., 2015), informações de sustentabilidade e rastreabilidade (KRIFLIK; YEATMAN, 2005), e informações nutricionais (ASCHEMANNWITZEL et al., 2013; NELSON et al., 2014).

A necessidade de personalização nutricional pode ser considerada um fator que influencia os consumidores em sua intenção de compra (BURTON et al., 2009), assim como a forma com que as informações nutricionais são apresentadas (BALTAS, 2001; BALCOMBE et al., 2016). A personalização nutricional pode ser constituída de acordo com fatores genéticos, histórico pessoal e estilo de vida (BOLAND, 2008). Os consumidores estão dispostos a pagar relativamente mais por informações nutricionais mais específicas quando comparado a rótulos com informações nutricionais genéricas (BALCOMBE et al., 2016). Entretanto, a personalização do produto tende a dificultar a confecção da tabela nutricional, principalmente em processos nos quais a previsão de valores dos componentes não é precisa.

A utilização de dispositivos que usufruem de tecnologia inteligente (smart technology) é um meio capaz de auxiliar os consumidores no momento da escolha dos produtos alimentícios (BALCOMBE et al., 2016), e possibilitam a customização de informações nutricionais (VOLKOVA et al., 2015). No entanto, qual a combinação de atributos dos dispositivos é mais indicada para fornecer as informações nutricionais de um alimento customizado? Este estudo possui o objetivo de identificar as funcionalidades de um dispositivo de geração de tabelas nutricionais de alimentos customizados, que agregam valor na visão do consumidor. Como contribuição, este estudo busca um direcionamento da pesquisa para composição de um dispositivo com a finalidade de fornecer informações nutricionais e de qualidade para o consumo de alimentos customizados.

\section{REFERENCIAL TEÓRICO}

\subsection{Tecnologias inteligentes disponíveis para reconhecimento do produto alimentício e mensuração de sua composição nutricional}

O elevado número de instalações de aplicativos disponibilizados para smartphones com funções de monitoramento nutricional, indica que há um interesse de consumo na utilização dessas tecnologias (FRANCO et al., 2016), também para fins alimentares. Esta tecnologia apresenta alternativas para que os usuários tenham a oportunidade de estabelecer um controle sobre hábitos de estilo de vida mais saudáveis (OKUMUS; BILGHAN, 2013) através do auto monitoramento de suas ações alimentares (HEBDEN et al., 2012), o que proporciona interpretação frequente dos dados auto observados e aprimora a personalização nutricional (MATILLA et al., 2012).

Na literatura, pesquisas possuem objetivos de identificar aplicativos e suas utilidades com a finalidade de fornecer benefícios nutricionais, em âmbitos de uma alimentação saudável 
(OKUMUS; BILGHAN, 2013; ARENS-VOLLAND et al., 2015; FRANCO et al., 2016) ou então direcionados a dietas restritivas destinadas a portadores de enfermidades como diabetes (DARBY et al., 2016), problemas renais (CAMPBELL; PORTER, 2015), câncer (BRUIN et al., 2015) e obesidade (WEARING et al., 2014). Alinhados aos aplicativos disponíveis para smartphones, pode-se também ser observado na literatura a utilização de dispositivos tecnológicos inteligentes que auxiliam no reconhecimento das características nutricionais dos alimentos e sua origem. Entre essas tecnologias, estudos identificam a utilização de etiquetas NFC combinadas com sistemas RFID (Radio Frequency Identification), tais como o NUTRIFLECT (REITBERGER et al., 2014) e o My Plate (CHEN et al., 2014); sistemas de reconhecimento de código de barras (ARENS-VOLLAND et al., 2015); sistemas de espectrometria de cores (ITUBE) (COSKUN et al., 2013), escâneres de espectrometria molecular (Food+Future Colab, TELLSPEC e SCIO Consumer Physics Rising) (ARENSVOLLAND et al., 2015; MAKARONA et al., 2016; VANDERROOST et al., 2017); e sistemas de reconhecimento de padrões de mastigação que permitem a entrada de voz para reconhecimento do alimento (BITBITE) (ARENS-VOLLAND et al., 2015).

Também verifica-se na literatura, diferentes tecnologias para a finalidade de identificação da qualidade de consumo dos alimentos tais como: etiquetas NFC com nanotubos de carbono (WANG et al., 2008), que respondem quanto a qualidade a partir da captação de gases e a interação com aplicativos de smartphone; sensores comestíveis e etiquetas com leitor óptico (NEETHIRAJAN; JAYAS, 2011), que analisam a presença de compostos provenientes da decomposição dos alimentos; e sensores comestíveis compostos de ouro e seda (TAO et al., 2012), que permitem a identificação do frescor dos alimentos através da interação com aplicativos de smatphones.

Podem ser encontrados no mercado, dispositivos com a finalidade de gerar informações sobre o alimento (reconhecimento de produto, reconhecimento de compostos alergênicos, mensuração da composição nutricional, personalização de dietas e verificação de qualidade). Diferentes formas de obtenção dessas informações podem ser verificadas, o que resulta em diferentes precisões de medidas e versatilidades. Isto pode ser observado em um exemplo, comparando-se os equipamentos WineTap (http://barkleymoonshot.tumblr.com/post/27503501953/more-moonshot-created-the-wine-

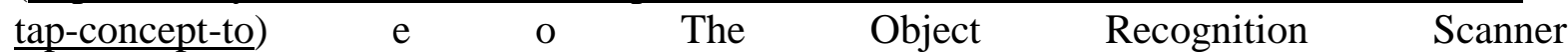
(http://mikarose.com/blog/post/toshiba_s_object_recognition_s/): possuem a mesma finalidade de reconhecimento do produto alimentício, entretanto enquanto o primeiro utiliza scanner para o reconhecimento de código de barras, o segundo utiliza sistemas de scanner para o reconhecimento dos padrões de cores de qualquer alimento. Além do reconhecimento do alimento, outras informações também podem ser geradas de forma diferente por diferentes dispositivos. Dispositivos como o $\quad$ MealSnap (http://www.appsafari.com/photos/16220/meal-snap-calorie-counting-magic/) ou $\quad$ o Im2calories (http://www.cv-foundation.org/openaccess/content_iccv_20), que retornam informações nutricionais a partir do reconhecimento de imagens, não possuem a mesma exatidão que dispositivos como o TellSpec (http://tellspec.com/en) que gera essas informações sobre a composição nutricional a partir do reconhecimento das moléculas presentes no alimento 


\section{METODOLOGIA}

\subsection{Procedimento de coleta de dados e Universo amostral experimental}

Foi realizada uma survey com a aplicação de um questionário on-line a partir da plataforma Qualtrics, por meio do qual obtiveram-se um total de 130 respostas válidas. Verificou-se a participação de respondentes de ambos os gêneros, com idades entre 19 e 59 anos. Os respondentes ainda foram classificados a partir de sua dieta restritiva ou não restritiva.

\subsection{Atributos e níveis}

Para a presente pesquisa, primeiramente, a partir de um grupo focado de 4 especialistas na área de Engenharia de produção, Engenharia de alimentos e nutrição, foram identificados e definidos 5 atributos que compõem o dispositivo em estudo, considerados como influentes na decisão de compra dos consumidores: (i) portabilidade e praticidade; (ii) precisão de medida; (iii) personalização de dietas; (iv) análise de qualidade do alimento; (v) preço. A Erro! Fonte de referência não encontrada. apresenta os atributos considerados para este estudo e seus respectivos níveis. Para a definição dos níveis de preço, foi realizada uma pesquisa de mercado prévia para que fosse possível a mensuração dos valores apresentados abaixo na Tabela 1.

Tabela 1. Atributos e seus níveis

\begin{tabular}{ll}
\hline Atributos & Níveis \\
\hline \multirow{2}{*}{ Portabilidade e praticidade } & Micro medidor embutido no celular \\
& Celular mais aparelho externo de medição \\
Precisão de medida & Alta precisão de medida \\
& Baixa precisão de medida \\
Personalização de dieta & Dietas personalizadas, específicas para cada indivíduo \\
& Dietas padronizadas para os indivíduos \\
Análise da qualidade do alimento & Presença do identificador de qualidade \\
& Ausência do identificador de qualidade \\
Preço* & Alto \\
& Baixo \\
\hline
\end{tabular}

Fonte: próprio autor

*Os níveis do preço estão aninhados a cada nível de precisão da medida:

Para alta precisão, \$378,75 - Preço nível alto e \$277,71 - Preço nível baixo;

Para baixa precisão, \$255,98 - Preço nível alto e \$124,94 - Preço nível baixo

\subsection{Projeto experimental e análise de dados}

$\mathrm{Na}$ área alimentícia, a utilização da metodologia de projetos experimentais e análise conjunta está relacionada a experimentos que verificam a interação entre diferentes componentes que constituem o produto alimentício e suas características (DECLOEDT et al., 2016), e também relacionada a experimentos que objetivam identificar a percepção dos consumidores perante diferentes combinações (cenários) que compõem produtos alimentícios (CICIA et al., 2012; BRUZZONE et al., 2015). Na literatura pode ser verificada a frequente presença da utilização de experimentos fatoriais fracionados (HEIDE et al., 2017; SCHERER et al., 2017). A partir dos atributos identificados, para este estudo foi realizado um projeto experimental fatorial fracionado $2^{5-1}$ com perfil completo (HAIR, 2005). A metodologia de análise conjunta baseada em escolhas (Choice-Based Conjoint Analysis) (CLARET et al., 2012; ASIOLI et al., 2016) foi aplicada, a partir da divisão dos 16 cenários em 4 blocos (LOUVIERE et al., 2000; MILLER, 2007) compostos por 4 cenários (combinações de atributos) em cada bloco. A partir da preferência de compra, os respondentes escolheram 1 cenário de cada bloco presente no experimento. Um último bloco ainda foi adicionado ao experimento composto por cada um dos 4 cenários escolhidos anteriormente, no qual o respondente, a partir de sua preferência, deveria escolher o melhor cenário de todos. Assim, foram consideradas participações válidas, 
somente quando o respondente forneceu 5 respostas, cada uma referente a um bloco presente no experimento. Para análise dos dados obtidos, foi utilizado o método de GLM (General Linear Model) a partir da estratégia de regressão logística (FRIEDMAN et al., 2000; HAIR, 2005).

\section{RESULTADOS}

A partir das 130 respostas consideradas válidas para este estudo, a metodologia de regressão logística foi utilizada considerando as 5 respostas pertinentes a cada respondente. A percepção de valor dos consumidores perante o dispositivo estudado nesta pesquisa, é uma função dependente de uma constante somada as variáveis representadas pelos atributos Portabilidade e praticidade (A); Precisão de medida (B); Personalização de dieta (C); Análise da qualidade do alimento (D); e Preço (E). Na Tabela 2 pode-se observar os valores obtidos para os coeficientes de cada atributo, assim como a constante da função que compõe a explicação da percepção de valor do consumidor. A Tabela 2 também apresenta os valores de significância referentes aos atributos.

Tabela 2. Apresentação dos coeficientes provenientes da regressão logística

\begin{tabular}{ccccccc}
\hline & Coeficientes & EP & Wald & gl & Sig. & Exp (coef.) \\
\hline Constante & $-3,433$ & 0,156 & 484,330 & 1 & 0,000 & 0,032 \\
A & 0,493 & 0,103 & 22,803 & 1 & 0,000 & 1,637 \\
B & 0,311 & 0,099 & 9,830 & 1 & 0,002 & 1,365 \\
C & 0,845 & 0,119 & 50,399 & 1 & 0,000 & 2,329 \\
D & 0,723 & 0,112 & 41,573 & 1 & 0,000 & 2,060 \\
E & $-0,512$ & 0,104 & 24,366 & 1 & 0,000 & 0,599 \\
\hline
\end{tabular}

Fonte: próprio autor

Como pode ser observado na Tabela 2, a constante e todos os atributos obtiveram significância a $1 \%$ partir da regressão linear $\left(p_{\text {valor }}<0,01\right)$ e assim, são significativos para a influência da percepção do consumidor. Quanto aos valores dos coeficientes mensurados a cada atributo, podemos verificar: (i) para o atributo "portabilidade e praticidade", o dispositivo composto com medidor embutido ao celular possui probabilidade 1,637 vezes maior de ser escolhido quando comparado ao dispositivo composto de medidor externo mais celular; (ii) para o atributo "precisão de medida", observa-se que dispositivos com alta precisão possuem probabilidade 1,365 vezes maior de serem escolhidos comparados a dispositivos que possuem baixa precisão; (iii) atributo "personalização de dieta" apresenta-se como a maior influência sobre a função de percepção de valor do consumidor, na qual dispositivos que fornecem dietas personalizadas possuem probabilidade 2,329 vezes maior de serem escolhidas, quando comparados aos dispositivos que fornecem dietas padronizadas; (iv) quanto ao atributo "análise de qualidade", a probabilidade de escolha de um dispositivo que possua o identificador de qualidade de consumo é 2,06 vezes maior quando comparado a dispositivos que não possuem essa função; (v) para o atributo preço, tem-se que a probabilidade de escolha de preços menores é aproximadamente 1,6 vezes maior quando comparados a cenários com valores de preços mais altos. A partir dos valores obtidos para os coeficientes, torna-se possível a definição da função de utilidade.

É necessário a verificação da necessidade de ajuste e qualidade do modelo de regressão logística obtido neste estudo. A partir da utilização da curva ROC, torna-se possível observar se a utilização da equação obtida no presente estudo promove resultados melhores quando comparado a equações aleatórias (HAIR, 2005). Os resultados obtidos para avaliação da curva ROC são apresentados na Tabela 3. Pode ser verificado que o modelo não necessita de ajuste, pois os resultados referentes as respostas da curva são significativos $\left(\mathrm{p}_{\mathrm{valor}}<0,001\right)$ e a integral da curva representa uma área superior a 0,7 . 
Tabela 3. Avaliação da curva ROC

\begin{tabular}{ccccc}
\hline & & & \multicolumn{2}{c}{ Intervalo de Confiança 95\% Assintótico } \\
\cline { 3 - 5 } Área & Erro Padrão & Sig. assintomática & Limite inferior & Limite superior \\
\hline 0,802 & 0,020 & 0,000 & 0,763 & 0,841 \\
\hline
\end{tabular}

Fonte: próprio autor

\section{CONCLUSÃO}

Foi obtida uma amostra heterogenia considerada representativa para possíveis consumidores. Os resultados revelam o interesse de $91 \%$ dos respondentes para aquisição do produto analisado. Com auxílio da função utilidade obtida, pode ser verificada a importância de cada atributo para o consumidor, e assim direcionar investimentos de produção. Neste estudo, foi possível observar que esta direção aponta para dispositivos: (i) que possuem micro medidor de informações nutricionais e de qualidade embutidos ao celular; (ii) que possuem alta precisão de medida; (iii) que fornecem informações nutricionais personalizadas, específicas para cada indivíduo; (iv) que possuem identificador de qualidade de consumo do produto alimentício; e (v) que possuem preço baixo. Futuros trabalhos poderiam abordar perspectivas de diferentes mercados consumidores.

\section{REFERÊNCIAS}

ARENS-VOLLAND, A. G.; SPASSOVA, L.; BOHN, T. Promising approaches of computersupported dietary assessment and management-Current research status and available applications. International Journal of Medical Informatics, v. 84, n. 12, p. 997-1008, 2015.

ASCHEMANN-WITZEL, J., GRUNERT, K. G., VAN TRIJP, H. C., BIALKOVA, S., RAATS, M. M., HODKGINS, C., ... \& KOENIGSTORFER, J. Effects of nutrition label format and product assortment on the healthfulness of food choice. Appetite, v. 71, p. 63-74, 2013.

ASIOLI, D., NæS, T., ØVRUM, A., \& ALMLI, V. L.. Comparison of rating-based and choice-based conjoint analysis models. A case study based on preferences for iced coffee in Norway. Food Quality and Preference, v. 48, p. 174-184, 2016.

BALCOMBE, K., FRASER, I., LOWE, B., \& MONTEIRO, D. S. 1. Information customization and food choice. American Journal of Agricultural Economics, v. 98, n. 1, p. 54-73, 2016.

BALTAS, G. Nutrition labelling: issues and policies. European Journal of Marketing, v. 35, n. 5/6, p. 708-721, 2001.

BOLAND, M. Innovation in the food industry: Personalised nutrition and mass customisation. Innovation, v. 10, n. 1, p. 53-60, 2008.

BRUZZONE, F., VIDAL, L., ANTÚNEZ, L., GIMÉNEZ, A., DELIZA, R., \& ARES, G.. Comparison of intensity scales and CATA questions in new product development: Sensory characterisation and directions for product reformulation of milk desserts. Food Quality and Preference, v. 44, p. 183-193, 2015.

BURTON, S., HOWLETT, E.; TANGARI, A. H.. Food for thought: How will the nutrition labeling of quick service restaurant menu items influence consumers' product evaluations, purchase intentions, and choices?. Journal of Retailing, v. 85, n. 3, p. 258-273, 2009.

CAMPBELL, J.; PORTER, J. Dietary mobile apps and their effect on nutritional indicators in chronic renal disease: A systematic review. Nephrology, v. 20, n. 10, p. 744-751, 2015. 
CHEN, P. H.; LIANG, Y. H.; LIN, T. C. Implementing a cooking and dietary management system using RFID technology. Mathematical Problems in Engineering, v. 2014, 2014.

CICIA, G.; DEL GIUDICE, T.; SCARPA, R. Consumers' perception of quality in organic food: a random utility model under preference heterogeneity and choice correlation from rank-orderings. British Food Journal, v. 104, n. 3/4/5, p. 200-213, 2002.

ClARET, A., GUERRERO, L., AGUIRRE, E., RINCÓN, L., HERNANDÉZ, M. D., MARTINEZ, I., ... \& RODRIGUEZ-RODRIGUEZ, C. Consumer preferences for sea fish using conjoint analysis: Exploratory study of the importance of country of origin, obtaining method, storage conditions and purchasing price. Food Quality and Preference, v. 26, n. 2, p. 259-266, 2012.

COSKUn, A. F., WONG, J., KHODADADI, D., NAGI, R., TEY, A., \& OZCAN, A. A personalized food allergen testing platform on a cellphone. Lab on a Chip, v. 13, n. 4, p. 636640, 2013.

DA SILVEIRA, G.; BORENSTEIN, D.; FOGLIATTO, F. S. Mass customization: Literature review and research directions. International Journal of Production Economics, v. 72, n. 1, p. $1-13,2001$.

DARBY, A., STRUM, M. W., HOLMES, E., \& GATWOOD, J.. A Review of Nutritional Tracking Mobile Applications for Diabetes Patient Use. Diabetes Technology \& Therapeutics, v. 18, n. 3, p. 200-212, 2016.

DE BRUIN, J., SCHUH, C., SEELING, W., LUGER, E., GALL, M., HUTTERER, E., ... \& SCHINDLER, K.. Assessing the feasibility of a mobile health-supported clinical decision support system for nutritional triage in oncology outpatients using Arden Syntax. Artificial Intelligence in Medicine, 2015.

DE PELSMAEKER, S.; DEWETTINCK, K.; GELLYNCK, X. The possibility of using tasting as a presentation method for sensory stimuli in conjoint analysis. Trends in Food Science \& Technology, v. 29, n. 2, p. 108-115, 2013.

DECLOEDT, A. I.; VAN LANDSCHOOT, A.; VANHAECKE, L Fractional factorial designbased optimisation and application of an extraction and UPLC-MS/MS detection method for the quantification of phytosterols in food, feed and beverages low in phytosterols. Analytical and Bioanalytical Chemistry, v. 408, n. 27, p. 7731-7744, 2016.

DIMARA, E.; SKURAS, D. Consumer demand for informative labeling of quality food and drink products: a European Union case study. Journal of Consumer Marketing, v. 22, n. 2, p. 90-100, 2005.

DURAY, R, WARD, P. T., MILIGAN, G. W., \& BERRY, W. L. Approaches to mass customization: configurations and empirical validation. Journal of Operations Management, v. 18, n. 6, p. 605-625, 2000.

FETTERMAnN, D. C.; ECHEVESTE, M. E. S.; MARTins, V. L. M.. Configurador de produto para a customização em massa na indústria automobilística. Produto \& Produção, v. 13 , n. 1, 2012.

FRANCO, R. Z., FALLAIZE, R., LOVEGROVE, J. A., \& HWANG, F. Popular NutritionRelated Mobile Apps: A Feature Assessment. JMIR mHealth and uHealth, v. 4, n. 3, 2016.

FRIEDMAN, J., HASTIE, T., \& TIBSHIRANI, R. Additive logistic regression: a statistical view of boosting (with discussion and a rejoinder by the authors). The annals of statistics, $\mathrm{v}$. 28, n. 2, p. 337-407, 2000. 
HAIR, Joseph F., ANDERSON, R. E., BABIN, B. J., \& BLACK, W. C. Multivariate Data Analysis: A global perspective. Upper Saddle River, NJ: Pearson, 2010.

HEBDEN, L.; CHEY, T.; ALLMAN-FARINELLI, M. Lifestyle intervention for preventing weight gain in young adults: a systematic review and meta-analysis of RCTs. Obesity Reviews, v. 13, n. 8, p. 692-710, 2012.

HEIDE, M.; OLSEN, S. O. Influence of packaging attributes on consumer evaluation of fresh cod. Food Quality and Preference, v. 60, p. 9-18, 2017.

KRIFLIK, L. S.; YEATMAN, Heather. Food scares and sustainability: a consumer perspective. Health, Risk \& Society, v. 7, n. 1, p. 11-24, 2005.

LINDLEY, D. Imperfect information for consumers. Consumer Policy Review, v. 17, n. 3, p. 74, 2007.

LOUVIERE, J. J.; HENSHER, D. A.; SWAIT, J. D. Stated choice methods: analysis and applications. Cambridge University Press, 2000.

MAKARONA, E., PETROU, P., KAKABAKOS, S., MISIAKOS, K., \& RAPTIS, I.. Pointof-Need bioanalytics based on planar optical interferometry. Biotechnology Advances, v. 34, n. 3, p. 209-233, 2016.

MATTILA, E., KORHONEN, I., SALMINEN, J. H., AHTINEN, A., KOSKINEN, E., SÃRELÃ, A., ... \& LAPPALAINEN, R. Empowering citizens for well-being and chronic disease management with wellness diary. IEEE Transactions on Information Technology in Biomedicine, v. 14, n. 2, p. 456-463, 2010.

MILLER, J. R. Attribute blocks: Visualizing multiple continuously defined attributes. IEEE Computer Graphics and Applications, v. 27, n. 3, p. 57-69, 2007.

NEETHIRAJAN, S.; JAYAS, D. S. Nanotechnology for the food and bioprocessing industries. Food and Bioprocess Technology, v. 4, n. 1, p. 39-47, 2011.

NELSON, M. E., LAYNE, J. E., BERNSTEIN, M. J., NUERNBERGER, A., CASTANEDA, C., KALITON, D., ... \& SINGH, M. A. F. The effects of multidimensional home-based exercise on functional performance in elderly people. The Journals of Gerontology: Series A, v. 59, n. 2, p. M154-M160, 2004.

OGAWA, S.; PILLER, F. T. Reducing the risks of new product development. MIT Sloan Management Review, v. 47, n. 2, p. 65, 2006.

OKUMUS, B.; BILGIHAN, A. Proposing a model to test smartphone users' intention to use smart applications when ordering food in restaurants. Journal of Hospitality and Tourism Technology, v. 5, n. 1, p. 31-49, 2014.

REITBERGER, W.; SPREICER, W.; FITZPATRICK, G. Situated and mobile displays for reflection on shopping and nutritional choices. Personal and Ubiquitous Computing, v. 18, n. 7, p. 1721-1735, 2014.

SCHERER, C.; EMBERGER-KLEIN, A.; MENRAD, K. Biogenic product alternatives for children: Consumer preferences for a set of sand toys made of bio-based plastic. Sustainable Production and Consumption, v. 10, p. 1-14, 2017.

SCHULDT, J. P. Does green mean healthy? Nutrition label color affects perceptions of healthfulness. Health Communication, v. 28, n. 8, p. 814-821, 2013. 
TAO, H., BRENCKLE, M. A., YANG, M., ZHANG, J., LIU, M., SIEBERT, S. M., ... \& KAPLAN, D. L. Silk-Based Conformal, Adhesive, Edible Food Sensors. Advanced Materials, v. 24, n. 8, p. 1067-1072, 2012.

VAN DER MERWE, D., KEMPEN, E. L., BREEDT, S., \& DE BEER, H. Food choice: student consumers' decision-making process regarding food products with limited label information. International Journal of Consumer Studies, v. 34, n. 1, p. 11-18, 2010.

VANDERROOST, M., RAGAERT, P., VERWAEREN, J., DE MEULANAER, B., DE BAETS, B., \& DEVLIEGHERE, F. The digitization of a food package's life cycle: Existing and emerging computer systems in the logistics and post-logistics phase. Computers in Industry, v. 87, p. 15-30, 2017.

VASILJEVIC, M.; PECHEY, R.; MARTEAU, T. M. Making food labels social: The impact of colour of nutritional labels and injunctive norms on perceptions and choice of snack foods. Appetite, v. 91, p. 56-63, 2015.

VOLKOVA, E.; MHURCHU, C. N. The influence of nutrition labeling and point-of-purchase information on food behaviours. Current Obesity Reports, v. 4, n. 1, p. 19-29, 2015.

WANG, F.; GU, H.; SWAGER, T. M. Carbon nanotube/polythiophene chemiresistive sensors for chemical warfare agents. Journal of the American Chemical Society, v. 130, n. 16, p. 5392-5393, 2008.

WEARING, J., NOLLEN, N., BEFORT, C., DAVIS, A. M., AGEMY, C. K. iPhone app adherence to expert-recommended guidelines for pediatric obesity prevention. Childhood Obesity, v. 10, n. 2, p. 132-144, 2014. 